\title{
Calcium intake from diet and supplement use during early pregnancy: the Expect study I
}

\author{
Jessica P. M. M. Willemse ${ }^{1}$ (D . Linda J. E. Meertens ${ }^{1} \cdot$ Hubertina C. J. Scheepers $^{2} \cdot$ Nina M. J. Achten ${ }^{3}$. \\ Simone J. Eussen ${ }^{1,4} \cdot$ Martien C. van Dongen ${ }^{1} \cdot$ Luc J. M. Smits $^{1}$
}

Received: 8 March 2018 / Accepted: 8 January 2019 / Published online: 19 January 2019

(c) The Author(s) 2019

\begin{abstract}
Purpose Adequate calcium intake during pregnancy is of major importance for the health of both mother and fetus. Up to date, evidence on the prevalence of inadequate calcium intake among pregnant women is sparse for Western countries, and it is unknown to what extent inadequate dietary calcium intake is adequately balanced by supplement use. The objective of this study was to estimate calcium intake from diet and supplement use during the early pregnancy in The Netherlands. Methods As part of the Expect cohort study, 2477 pregnant women (8-16 weeks of gestation) completed an online questionnaire including questions on baseline characteristics, the use of calcium containing supplements, and a short food-frequency questionnaire (FFQ). Intake data were used to calculate median calcium intakes from diet, from supplements, and combined, and to compare these values with currently accepted requirement levels.

Results Forty-two percent of the pregnant women had a total calcium intake below the estimated average requirement of $800 \mathrm{mg} /$ day. Median (interquartile range) calcium intake was 886 (611-1213) mg/day. Calcium or multivitamin supplements were used by $64.8 \%$ of the women at 8 weeks of gestation, with a median calcium content of $120.0(60.0-200.0) \mathrm{mg} / \mathrm{day}$. Prenatal vitamins were the most often used supplements $(60.6 \%)$.

Conclusions Forty-two percent of Dutch pregnant women have an inadequate calcium intake. Supplements are frequently used, but most do not contain sufficient amounts to correct this inadequate intake.
\end{abstract}

Keywords Calcium intake $\cdot$ Pregnancy $\cdot$ Diet $\cdot$ Supplements $\cdot$ Dairy products $\cdot$ Prenatal vitamins

\section{Introduction}

Fetal growth places high demands on maternal calcium status $[1,2]$. Although part of the demand is met by means of increased intestinal calcium absorption [3-5], adequate calcium intake by the mother remains important. Insufficient

Jessica P. M. M. Willemse

jessica.willemse@maastrichtuniversity.nl

1 Department of Epidemiology, CAPHRI Care and Public Health Research Institute, Maastricht University, PO Box 616, 6200 MD Maastricht, The Netherlands

2 Department of Obstetrics and Gynaecology, Maastricht University Medical Centre, PO 5800, 6202 AZ Maastricht, The Netherlands

3 Maastricht University, PO 616, 6200 MD Maastricht, The Netherlands

4 CARIM School for Cardiovascular Diseases, Maastricht University, Maastricht, The Netherlands calcium intake poses risks to both fetus and mother. Fetal risks include restricted intrauterine growth, low birth weight, poor bone mineralization, and preterm birth, whereas maternal risks include hypertension and preeclampsia $[6,7]$. Several trials have shown the beneficial effects of calcium supplementation in the prevention of preeclampsia [8-12].

Recommended calcium intake varies between countries from 900 to $1200 \mathrm{mg} /$ day [13, 14]. The World Health Organization (WHO) and the Food and Agriculture Organization (FAO) of the United Nations recommend a dietary intake of $1200 \mathrm{mg} /$ day of calcium for pregnant women and $1000 \mathrm{mg} /$ day for non-pregnant adults (19-50 years old) [5, 15]. In The Netherlands, the recommended dietary allowance (RDA) is $1000 \mathrm{mg} /$ day for all adults, irrespective of pregnancy status [16].

Diet is the main contributor to total calcium intake [17]. Large differences in calcium intakes between countries have been linked with diversity in food habits and access to food [18]. A WHO survey showed dietary calcium intake to be 
inadequate $(<1000 \mathrm{mg} /$ day $)$ among $89 \%$ of nulliparous pregnant women in developing countries [18]. In view of the good availability and affordability of dairy products in The Netherlands, calcium intake might be expected to be adequate, and likewise in other Western countries. Nevertheless, in a previous Dutch study among pregnant women, mean dietary calcium intake of Dutch pregnant women was found to be just above $1100 \mathrm{mg} /$ day with a standard deviation of 311 [19], indicating that, assuming a normal distribution, one in every six women may have an intake below the estimated average requirement of $800 \mathrm{mg} /$ day [20].

Dietary calcium intake can be complemented with the intake of calcium-containing food supplements. The WHO currently recommends calcium supplementation as part of antenatal care for women with an inadequate dietary calcium intake to lower the risk of developing preeclampsia [5]. To our knowledge, only a few studies analyzed total daily calcium intake from both food and supplement use [21, 22]. Moreover, these studies did not evaluate a large cohort in a Western country. It is also unclear to what extent calcium is advised or prescribed in Dutch clinical practice or how much elementary calcium is ingested from both diet and supplements by Dutch pregnant women. In a small survey, we found that $89 \%$ of the gynecologists $(n=18)$ and $10 \%$ of the midwives $(n=30)$ counsel their patients on calcium supplements. Most counseling (74\%) was directed, toward pregnant women at high risk for preeclampsia.

The main purpose of this study was to estimate total daily calcium intake from both food and supplement use among Dutch women during the early pregnancy. To this end, we used data from a population-based pregnancy cohort. Our study's second purpose was to evaluate the calcium content of currently used supplements.

\section{Subjects and methods}

\section{Study population}

Data were collected as part of the Expect study I, a prospective cohort study performed in the south-eastern part of the Netherlands with the purpose of validating a number of firsttrimester obstetric prediction models. Pregnant women were recruited in 36 midwifery practices and 6 hospitals between July 2013 and January 2015, with follow-up until December 2015. Eligibility criteria for the Expect study were: less than 16 weeks of gestation and a minimum age of 18 years. The Medical Ethical Committee of the Maastricht University Medical Centre evaluated the study protocol and declared that no ethical approval was necessary (MEC 13-4-053). All participating women gave online informed consent.

Figure 1 shows the flowchart of the study population. For this study, we included only women that filled out the questionnaire at 8 weeks of gestation or later, allowing most women to have adapted their diet or started using supplements. On the basis of this restriction, 136 women had to be excluded. We also excluded one woman with the missing values in all dietary questions. Finally, 2477 women were available for analysis.

\section{Data collection}

Women were asked to complete an online questionnaire before 16 weeks of gestation (or a paper version if requested), containing questions about a range of variables including socio-demographic characteristics, lifestyle, obstetric history, medical conditions, and family history. Supplement use before and during pregnancy was asked for, focusing on folic acid, vitamin D, prenatal vitamins, general multivitamins, and calcium preparations, as well as dietary intake of calcium and vitamin D.

\section{Dietary intake}

The Dutch FFQ-TOOL ${ }^{\mathrm{TM}}$ (FFQ = food-frequency questionnaire) was used for the selection of food products contributing to calcium and vitamin D intake [21]. For the present study, we only focused on calcium intake. Based on food and nutrient intake data for 20-45 year old (non-pregnant) women who participated in the Dutch National Food Consumption Survey 2007-2010 (DNFCS 2007-2010) and food composition data from the Dutch Food Composition (NEVO) Table 2010, which were embedded in the Dutch FFQ-TOOL ${ }^{\text {TM }}$, we made the FFQ-TOOL select those food products that cumulatively covered $>80 \%$ of the variance in calcium intake [23]. The selection procedure resulted in 18 food items, for which both the frequency of use (reference period: last month) and the average daily amount of use were asked: milk and buttermilk; yoghurt and fromage frais (with or without fruit); yoghurt drinks and other dairy beverages; chocolate milk; custard and pudding; Dutch cheese; nonDutch cheese and cream cheese; cheese spread; bread spread (sub types: margarine; low-fat margarine; and butter); cooking fat (bake and fry products); and fish (subtypes: fat fish such as salmon, mackerel, eel, and white harring; lean fish such as codfish, tilapia, panga fish and trout; white fish filet; smoked or steamed fish; herring; and fish fingers. Milk, milk products, and cheese were included in the questionnaire as major sources of calcium, bread spread, bake and fry products, and fish as major sources of vitamin D.

We chose to include questions on food products that cumulatively cover $>80 \%$ of the variance in calcium intake and eliminate food products with small calcium contents. This consideration was made to encourage participants to complete the full questionnaire of the Expect study I. Covering the complete dietary calcium intake in this study was not 
Fig. 1 Inclusion flowchart

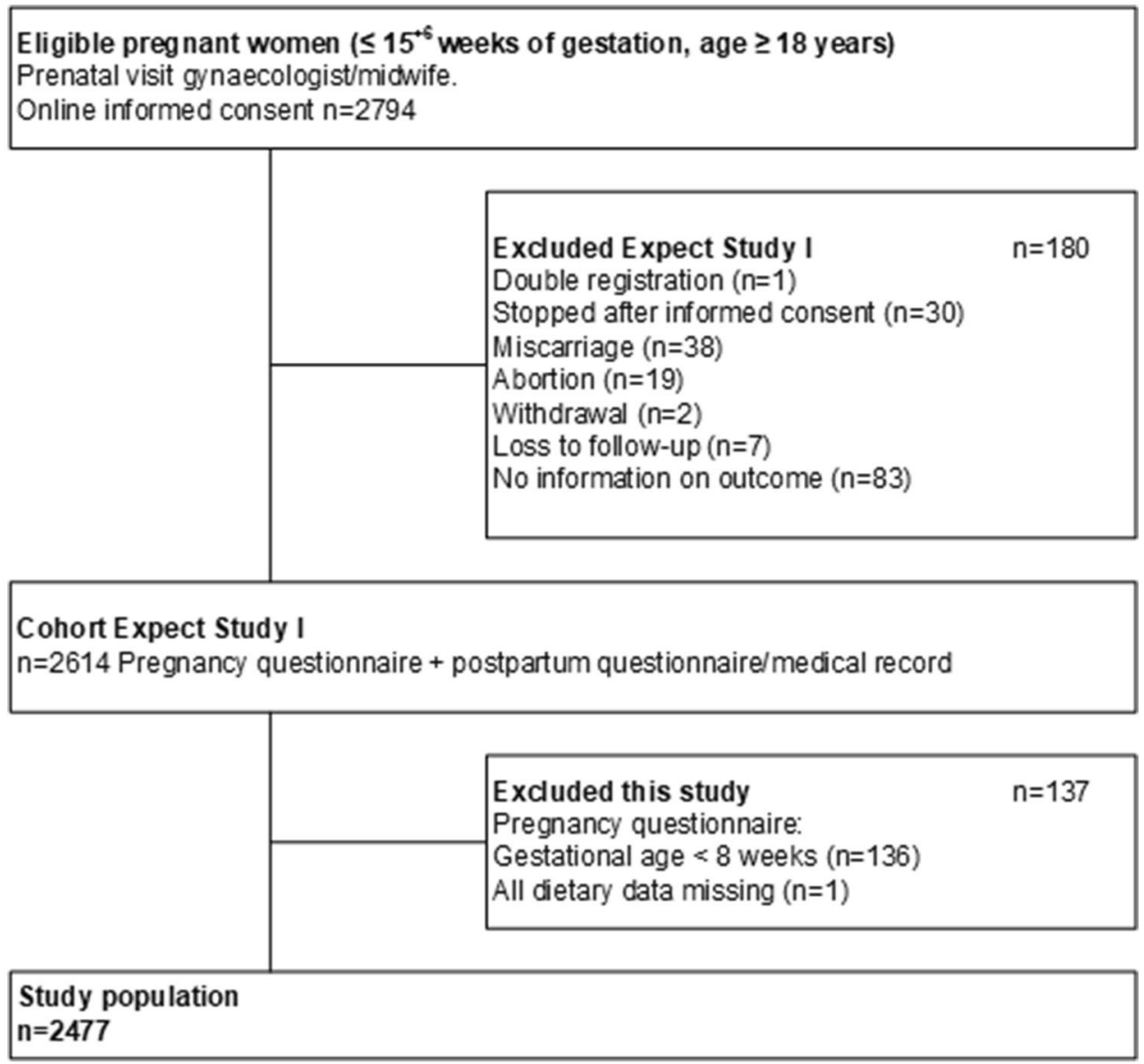

feasible, as the FFQ was part of an intensive questionnaire containing several pregnancy-related topics, with the purpose of validating a number of first-trimester obstetric prediction models. The food items in the questionnaire covered an estimated $62 \%$ of total absolute dietary calcium intake. In case dietary calcium intakes were either $0 \mathrm{mg} /$ day or over $1750 \mathrm{mg} /$ day, women were contacted to check whether any unintended errors were made. In the group of women with dietary intakes above the tolerable upper level of $2500 \mathrm{mg}$ per day, four women reported correct intakes and the rest made an unintended error, which was corrected after contact with the participant. Since intakes $<200 \mathrm{mg}$ calcium in the mostly Caucasian population of this study are relatively low, we checked whether exclusion of these outliers (dietary calcium intake $<200 \mathrm{mg}, n=106$ ) affected median dietary calcium intake and the percentage of total inadequate intake in an extra-sensitivity analysis.

\section{Supplements}

Questions on potential calcium-containing supplements such as prenatal vitamins, general multivitamins, and calcium supplements, were included for this study. We requested time and period of use (start of usage before and during pregnancy, when potentially stopped, current use), brand and any subtype, frequency of use per week, and amount of tablets per day.

Calcium was standardized to the elemental form in milligrams, based on the labels. We contacted the manufacturers for the clarification when the exact elementary amount of calcium in the supplement was unclear.

\section{Data analysis}

Baseline characteristics were analyzed and presented as percentages. Missing values in the baseline characteristics of the Expect I cohort regarding education level $(n=3)$ and body mass index (BMI) $(n=5)$, were imputed using stochastic regression imputation based on predictive mean matching [24].

We calculated individual daily dietary calcium intake by multiplying frequency of consumption by consumed amounts of all the assessed food products and combining product intake (grams per day) with calcium content of each product according to the Dutch Food Composition Table of 2010 (NEVO-online 2010) [25] and DNFCS2007-2010 [23]. Missing frequency and amount values were imputed with the modal value of all the valid values for the specific 
variable. To account for the incomplete coverage of the foodfrequency questionnaire, we adjusted the estimated calcium intake values (estimated intake*100/61.65). In this way, adjusted total calcium intakes were used in the analyses and presented in the results.

We calculated percentages of women using the different calcium-containing supplements for each week of gestation. We calculated daily calcium intake from supplement use by combining frequency, amount of supplements, and content of specific supplements among current users at 8 weeks of gestation. In case a participant used a supplement but did not know the exact (subtype) brand, the modal value was imputed. Median values of calcium supplement intake at 8 weeks of gestation were calculated.

Median values of total calcium intake, dietary calcium intake, and calcium intake from supplement use were calculated and presented in milligrams per day, with interquartile range (IQR). Total calcium intake was compared to the Recommended Dietary Allowance (RDA) of $1000 \mathrm{mg} /$ day and the Estimated Average Requirement (EAR) of $800 \mathrm{mg} / \mathrm{day}$. An intake level of $800 \mathrm{mg}$ calcium is expected to satisfy the needs of $50 \%$ of all pregnant women [26, 27]. The EAR cut point method proposed by the Institute of Medicine (IOM) was used to assess the level of inadequacy in calcium intake in our population $[20,28]$.

Analyses were performed using IBM SPSS statistics version 23

\section{Results}

\section{Study population}

The baseline characteristics of the study population $(n=2477)$ are shown in Table 1. Almost all women were of Caucasian origin, more than $80 \%$ of the women were aged 26-35 years, and 55\% had a finished tertiary level of education. Half of the study population was nulliparous and 54\% of the women had a normal prepregnancy BMI (20.0-24.9).

\section{Total calcium intake}

Median (IQR) calcium intake was 886 (611-1213) mg/day. Forty-two percent of the women $(n=1045)$ had a total calcium intake below the EAR and 60\% $(n=1489)$ did not meet the RDA of $1000 \mathrm{mg}$ calcium per day (Fig. 2).

\section{Dietary calcium intake}

Diet was the main contributor to total calcium intake (Fig. 2). Almost all women (99.8\%) used calcium-containing dietary products. Dietary calcium intake varied from a minimum of $0 \mathrm{mg}$ to a maximum of $4215.8 \mathrm{mg}$ calcium
Table 1 Baseline characteristics of the study population

\begin{tabular}{ll}
\hline Characteristics & $N=2477^{\mathrm{a}}(\%)$ \\
\hline Age (years) & 11 \\
$18-25$ & 43 \\
$26-30$ & 38 \\
$31-35$ & 9 \\
$>35$ & \\
BMI before pregnancy & 13 \\
$<20.0$ & 54 \\
$20.0-24.9$ & 22 \\
$25.0-29.9$ & 11 \\
$>30.0$ & \\
Ethnicity & 97 \\
Caucasian & 0.8 \\
Asian & 0.1 \\
Negroid & 0.4 \\
Hispanic & 2 \\
Mixed & \\
Parity & 51 \\
Nulliparity & \\
Level of education & 55 \\
Tertiary & \\
\hline &
\end{tabular}

${ }^{\text {a }}$ Percentages do not always add up to $100 \%$ due to rounding

per day. Median (IQR) dietary calcium intake was $798 \mathrm{mg} /$ day (530-1113). Most contributing to dietary calcium intake were consumption of Dutch cheese (31\%) and milk (27\%).

Based on just food intake, $50 \%$ of the women had an intake below the EAR of $800 \mathrm{mg} /$ day. In this group, $16 \%$ added enough calcium from supplements to their diet to reach the EAR.

\section{Supplement use}

Supplement use per category varied before and during early pregnancy (Fig. 3). Before pregnancy, 29\% of the women started the use of prenatal vitamins, and at 8 weeks of gestation, this type of supplement was used by $61 \%$. On the other hand, the use of general multivitamins declined from $8 \%$ usage before pregnancy to $5 \%$ at 8 weeks of gestation. The use of specific calcium supplements did not show much variation. Supplements containing only calcium were used by only $1 \%$ of the women before pregnancy and $2 \%$ used them at 8 weeks of gestation.

\section{Calcium content of supplements}

Median (IQR) calcium intake from supplement use at 8 weeks of gestation was $120 \mathrm{mg}$ / day among users (60-200).

The median (IQR) calcium intake from prenatal vitamins at 8 weeks of gestation (61\%) was $120 \mathrm{mg} /$ day (60-200). 
Fig. 2 Calcium intake from diet and supplement use at 8 weeks of gestation

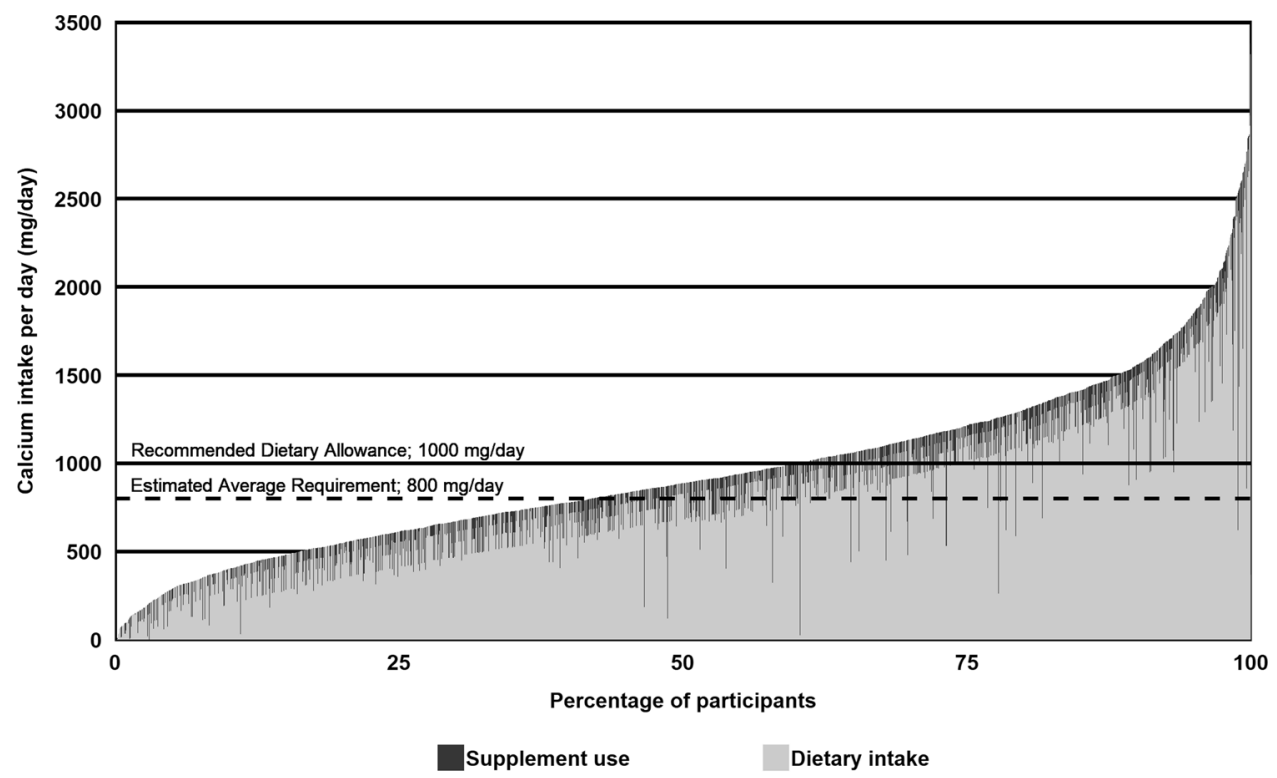

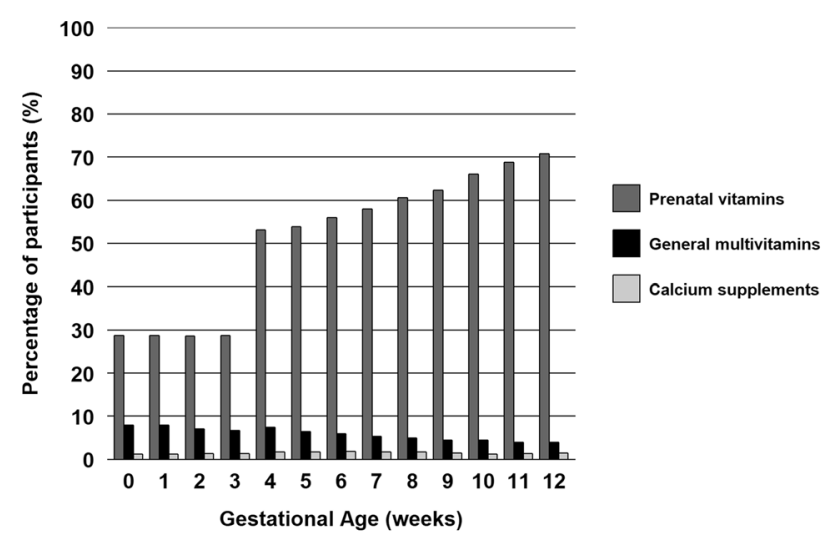

Fig. 3 Calcium-containing supplement use per gestational age

The calcium content of various calcium-containing prenatal vitamins ranged from 60 to $326 \mathrm{mg}$ calcium per tablet. Three of twenty-three prenatal vitamins did not contain calcium.

For general multivitamins, the median (IQR) calcium intake was $103 \mathrm{mg} /$ day (5-162) at 8 weeks of gestation. The content of the 66 reported general multivitamins ranged from 10 to $500 \mathrm{mg}$ per tablet and 18 reported general multivitamins did not contain any calcium.

The median (IQR) calcium intake from calcium supplements at 8 weeks of gestation (2\%) was $337 \mathrm{mg} /$ day (214-508). The content of reported calcium supplements ranged from 240 to $1000 \mathrm{mg}$ calcium per tablet.

\section{Sensitivity analysis}

Exclusion of participants with dietary calcium intakes $<200 \mathrm{mg}(n=106)$ resulted in a median (IQR) dietary calcium intake of 824 (570-1129) mg and a median (IQR) total calcium intake of 906 (649-1229) mg per day. The percentage of women with a total calcium intake below the EAR was $40 \%$, differing not much from the percentage in the total population.

\section{Discussion}

\section{Main finding}

We found that $42 \%$ of the population had a total calcium intake below the EAR of $800 \mathrm{mg}$ calcium per day.

\section{Previous findings on calcium intake}

The beneficial effects of an adequate calcium intake during pregnancy have been a topic of interest for quite a long time [6, 7, 29-35]. Dietary calcium intake during pregnancy has been mainly assessed among populations in the developing countries, and lowest intakes were predominantly found in Asia and Africa [18]. A WHO systematic review from 2005 showed that the average dietary calcium intake in developed countries was below the RDA in half of the included studies [18]. More recent studies showed even higher proportions of inadequate calcium intake in women of reproductive age in the developed countries with inadequacy in more than half of the population [31, 32].

Two previous studies have looked at calcium intake among women in The Netherlands. Among pregnant women from Rotterdam, mean dietary calcium level in the first trimester of pregnancy was found to be greater than results of our current study show, namely 1108 (sd, 311) [18]. 
Assessment of dietary calcium intakes in this study was done more extensively, by means of a modified FFQ containing 293 food items. On the assumption of a normal distribution of calcium intakes, these figures would indicate that about one in every six women would have an intake below EAR, which is a lower proportion than found in our study. However, as observed in our study, intakes may be strongly right-skewed, implying a higher proportion of women with sub-EAR calcium intakes. Furthermore, the women included in the Rotterdam study had a more diverse ethnicity and data on supplement use are lacking.

In the Dutch National Food Consumption Survey 2007-2010, more than one-fourth of the women of reproductive age (age categories 19-30 and 31-50 years) did not reach the EAR of $800 \mathrm{mg}$ calcium per day by diet alone. Calcium-containing supplements were used by 4-8\% [23]. These women were, however, from the general (non-pregnant) population, while pregnancy may cause nausea and changes in food choices [36], possibly explaining the lower intake in our population. Next to this, while we cover $62 \%$ of total absolute dietary calcium intake with our inquired food items, the Food Consumption Survey included a more precise method, using two non-consecutive 24-h dietary recalls. The differences in population and methods may have contributed to the $<17 \%$ difference in the amount of women with inadequate intake.

Even though the used assessments for dietary calcium intake in the previously mentioned studies were more detailed and perhaps more accurate, our study points into the important direction of an inadequate calcium intake in a Western population. In addition, to our knowledge, the current study is the first one that assessed the complete calcium intake from both diet and supplement use in Dutch pregnant women.

\section{Implications}

We showed that dietary sources alone are insufficient to meet an adequate calcium intake in $50 \%$ of the pregnant women. Ingestion of the amount of calcium necessary to reach the EAR is not easily achieved via diet alone [31]. Although dairy products are the largest contributor to calcium intake, $100 \mathrm{~g}$ milk contains only 108-200 mg calcium [25]. Changing dietary habits is challenging for many women despite a presumed higher awareness of health issues during pregnancy [37, 38].

Dietary intake can be complemented by supplements to achieve an adequate calcium intake. Supplement use will result in a higher prevalence of an adequate calcium intake $[32,39]$. In the current study, $74 \%$ of our participants used multivitamin or calcium supplements. Morisset et al. [40] demonstrated calcium-containing supplements are being used by $73 \%$ of their study population $(n=1186)$, which is comparable to our study population. However, the calcium content of the most frequently used supplements-prenatal vitamins - is insufficient to complement the dietary calcium intake to a level that meets EAR. Remarkably, 3 of the 23 used prenatal vitamins did not contain any calcium. Moreover, the effectiveness of prenatal vitamins other than separate folic acid and vitamin D supplements has never been proven [41]. Because of the insufficient calcium content of most prenatal vitamins and multivitamins, it seems essential to advise the use of separate calcium supplements for pregnant women with an inadequate calcium intake. We recently showed that advising all pregnant women to use calcium supplements can be expected to cause substantial reductions in the incidence of preeclampsia as well as related health care costs [42]. No major side effects have been described, but the tolerable upper level of $2500 \mathrm{mg}$ calcium per day should be taken into account, since hypercalcemia could cause renal insufficiency, vascular and soft-tissue calcification, hypercalciuria, and kidney stones [20, 23].

\section{Strengths and limitations}

Our study provides an overview of calcium intake levels by pregnant women based on not just diet but also supplement use. A major strength of our study is the large sample size of 2477 participants. In our population, women of Caucasian origin were somewhat overrepresented and more than half of the population had a high educational level, which corresponds to the composition of the population of Dutch women in their thirties [43]. A previous Canadian study showed that calcium intake was lower in women with lowest educational levels [40], so the overrepresentation of high educated women in our study may have led to an underestimation of the total percentage of women with an inadequate intake.

A few limitations of our study should be addressed. First, since there is no biochemical assay to display the nutritional calcium status, we had to depend on questionnaires. Repeated dietary recalls or records might have been considered as more accurate approaches for food intake assessment. However, this method would not be achievable in a large cohort. Nevertheless, the FFQ method is widely used for food product and nutrient intake assessment, and although its main strength is in the ranking of individuals according to their intakes of frequently used foods and nutrients, it is also considered a feasible tool to gain insight in the percentage of inadequate intake in a large population [44]. Second, our assessment of dietary calcium intake included only those products with the highest contribution to their calcium content to minimize the load of the questionnaire. Moreover, the selected products inquired in our questionnaire contributed to more than $60 \%$ of all dietary calcium intake and we recalculated 
total calcium intake to $100 \%$ [45]. This selection procedure probably has resulted in an underestimation of dietary calcium intake, requiring adjustment [40]. The five most contributing food products to dietary calcium intake that were not inquired in our study were bread (3.9\%), water $(3.7 \%)$, cooked or stir fried vegetables $(3.5 \%)$, coffee $(2.2 \%)$, and tea (1.6\%). Even though the contribution of these food products to total calcium intake is limited and already covered in the recalculation from 61.65 to $100 \%$, we compared our methods to the methods used in a more extensive FFQ. To ensure that our methods estimated the correct total calcium amount, we applied our methods to the data of pregnant women from an older birth cohort study $(N=2855)$ [46]. Thirty-nine from the 213 food items from the KOALA-FFQ were comparable to the food items which we inquired in our study. Total calcium intake based on the 39 food items was strongly correlated to calcium intake based on the complete KOALA-FFQ (Pearson's $r=0.95$ ). Women who did not consume food products inquired in our questionnaire were contacted and have a recalculated total dietary calcium intake of $0 \mathrm{mg}$, while they may have consumed other calcium-containing products. However, sensitivity analysis did not show large differences after exclusion of outliers. Third, recall may not have been optimal as dietary intake was inquired for the month prior to filling in the questionnaire. Finally, there may have been intra-individual variation in food intake which was not covered by the applied measurement procedure. Food intake may vary over time and, perhaps, especially during pregnancy as women may experience sickness in the early pregnancy. However, the previous evidence showed that calcium intake from supplement use does not differ much across trimesters [47].

\section{Conclusion}

Our study provides insight in calcium intake from both diet and supplement use during the early pregnancy. We found that $42 \%$ of the pregnant population had an inadequate total calcium intake. Efforts to reach an adequate calcium intake are recommendable for all women with inadequate calcium intakes. We advise further research into barriers and facilitators for obtaining an adequate calcium intake during pregnancy. In addition, research into risk indicators for an inadequate calcium intake could be useful to implement directed health promotion to target groups.

Acknowledgements We are grateful to all women who participated in the Expect study. We acknowledge participant recruitment by the participating midwifery practices and departments of obstetrics and gynaecology of hospitals in the Province of Limburg. We also acknowledge
Nicole Wijckmans, Department of Epidemiology, Maastricht University, for selecting the food items for the questionnaire.

Author contribution LJEM, HCJS, and LJMS contributed to the conception and design of the Expect study I. LJEM was responsible for the data collection. SJE and MCD were responsible for the selection of food items (FFQ) and assisted in the analysis of dietary intake data. JPMMW conducted the statistical analyses, interpreted the data, and drafted the manuscript. All authors were involved in interpretation of the outcomes, critically reviewed draft versions, and approved the final manuscript.

Funding The Expect study was funded by The Netherlands Organization for Health Research and Development, Pregnancy and Childbirth program (ZonMw Grant 209020007). The funding organization had no role in the design and conduct of the study, analysis or interpretation of data, decision to publish, or preparation of the manuscript.

\section{Compliance with ethical standards}

Conflict of interest The authors declare that they have no conflicts of interest.

OpenAccess This article is distributed under the terms of the Creative Commons Attribution 4.0 International License (http://creativeco mmons.org/licenses/by/4.0/), which permits unrestricted use, distribution, and reproduction in any medium, provided you give appropriate credit to the original author(s) and the source, provide a link to the Creative Commons license, and indicate if changes were made.

\section{References}

1. Ward KA, Adams JE, Mughal MZ (2005) Bone status during adolescence, pregnancy and lactation. Curr Opin Obstet Gynecol 17(4):435-439

2. Ritchie LD et al (1998) A longitudinal study of calcium homeostasis during human pregnancy and lactation and after resumption of menses. Am J Clin Nutr 67(4):693-701

3. Kalkwarf HJ, Specker BL (2002) Bone mineral changes during pregnancy and lactation. Endocrine 17(1):49-53

4. Cross NA et al (1995) Calcium homeostasis and bone metabolism during pregnancy, lactation, and postweaning: a longitudinal study. Am J Clin Nutr 61(3):514-523

5. World Health Organisation (2013) Guideline: calcium supplementation in pregnant women. Geneva, World Health Organization http://apps.who.int/iris/bitstream/10665/85120/1/9789241505 376_eng.pdf. Assessed 6 Jun 2016

6. Crowther CA et al (1999) Calcium supplementation in nulliparous women for the prevention of pregnancy-induced hypertension, preeclampsia and preterm birth: an Australian randomized trial. FRACOG and the ACT Study Group. Aust N Z J Obstet Gynaecol 39(1):12-18

7. Bucher HC et al (1996) Effect of calcium supplementation on pregnancy-induced hypertension and preeclampsia: a meta-analysis of randomized controlled trials. JAMA 275(14):1113-1117

8. Hofmeyr GJ, Lawrie TA, Atallah AN, Duley L, Torloni MR (2014) Calcium supplementation during pregnancy for preventing hypertensive disorders and related problems. Cochrane Database Syst Rev (6):1-74 
9. Patrelli TS et al (2012) Calcium supplementation and prevention of preeclampsia: a meta-analysis. J Matern Fetal Neonatal Med 25(12):2570-2574

10. Villar J, Belizan JM, Fischer PJ (1983) Epidemiologic observations on the relationship between calcium intake and eclampsia. Int J Gynaecol Obstet 21(4):271-278

11. Tang R et al (2015) Limited evidence for calcium supplementation in preeclampsia prevention: a meta-analysis and systematic review. Hypertens Pregnancy 34(2):181-203

12. Villar J et al (2003) Nutritional interventions during pregnancy for the prevention or treatment of maternal morbidity and preterm delivery: an overview of randomized controlled trials. J Nutr 133(5 Suppl 2):1606S-1625S

13. Ross AC, Taylor CL, Yaktine AL, Del Valle HB (2011) Dietary reference intakes for calcium and vitamin D. National Academy of Sciences, Washington DC

14. EFSA Panel on Dietetic Products, Nutrition and Allergies (2015) Scientific opinion on dietary reference values for calcium. EFSA J 13(5):4101

15. Food and Agriculture Organisation (2001) Human vitamin and mineral requirements. http://www.fao.org/3/a-y2809e.pdf. Assessed 6 Jun 2016

16. Hart W (2000) Recommendations for calcium and vitamin D in the report 'Food standards' of the health council. Ned Tijdschrift Tandheelkunde 144(110):78-81

17. Flynn A et al (2009) Intake of selected nutrients from foods, from fortification and from supplements in various European countries. Food Nutr Res. https://doi.org/10.3402/fnr.v53i0.2038

18. Merialdi M, Mathai M, Ngoc NTN, Purwar M, Campodonico L, Abdel-Aleem H, Hofmeyr GJ, Rojas MX, Perez-Cuevas R, Joseph G, Hieu NT, Radha NA, Motghare V, Carroli G, Cerro del S, Giordano D, Ahmed A, Mangesi L, Roodt A, Mahmoud FA, Piaggio G, Villar J (2005) World Health Organisation systematic review of the literature and multinational nutritional survey of calcium intake during pregnancy. Fetal Matern Med Rev 16(02):97-121

19. Heppe DH et al (2013) Maternal first-trimester diet and childhood bone mass: the generation R study. Am J Clin Nutr 98(1):224-232

20. Ross AC (2011) The 2011 report on dietary reference intakes for calcium and vitamin D. Public Health Nutr 14(5):938-939

21. Cormick $\mathrm{G}$ et al (2014) Gaps between calcium recommendations to prevent pre-eclampsia and current intakes in one hospital in Argentina. BMC Res Notes 7:920

22. Jia HX et al (2016) Mineral intake in urban pregnant women from base diet, fortified foods, and food supplements: focus on calcium, iron, and zinc. Biomed Environ Sci 29(12):898-901

23. Van Rossum CTM, Fransen HP, Verkaik-Kloosterman J, BuurmaRethans EJM, Ocké MC (2006) Dutch national food consumption survey 2007-2010; diet of children and adults aged 7 to 69 years. Bilthoven: Ministry of Health, Welfare and Sport, National Institute for Public Health and the Environment. http://www.rivm. nl/dsresource?objectid=3f528864-36cb-400b-8411-a47db4f99d f4\&type $=$ org \&disposition=inline Assessed 6 Jun 2016

24. Van Buuren S (2012) Flexible imputation of mising data. Chapmann and Hall/CRC, Boca Raton

25. Dutch National Institute for Public Health and the Environment (RIVM) (2010) NEVO-online 2010-2.0. RIVM, Bilthoven

26. Ross AC et al (2011) The 2011 dietary reference intakes for calcium and vitamin D: what dietetics practitioners need to know. $\mathrm{J}$ Am Diet Assoc 111(4):524-527

27. Institute of Medicine (US) Standing Committee on the Scientific Evaluation of Dietary Reference Intakes (1997) Dietary reference intakes for calcium, phosphorus, magnesium, vitamin D, and fluoride. National Academy of Sciences, Washington DC

28. Institute of Medicine (2000) Dietary reference intakes: applications in dietary assessment. The National Academies Press, Washington DC. https://doi.org/10.17226/9956
29. Jarjou LM et al (2010) Effect of calcium supplementation in pregnancy on maternal bone outcomes in women with a low calcium intake. Am J Clin Nutr 92(2):450-457

30. Sabour $\mathrm{H}$ et al (2006) Relationship between pregnancy outcomes and maternal vitamin D and calcium intake: a cross-sectional study. Gynecol Endocrinol 22(10):585-589

31. Nicklas TA, O'Neil CE, Fulgoni VL (2009) The role of dairy in meeting the recommendations for shortfall nutrients in the American diet. J Am Coll Nutr 28(Suppl 1):73s-81s

32. Bailey RL et al (2010) Estimation of total usual calcium and vitamin D intakes in the United States. J Nutr 140(4):817-822

33. Melnik BC, John SM, Schmitz G (2015) Milk consumption during pregnancy increases birth weight, a risk factor for the development of diseases of civilization. J Transl Med 13:13

34. Heppe DH et al (2011) Maternal milk consumption, fetal growth, and the risks of neonatal complications: the generation $\mathrm{R}$ study. Am J Clin Nutr 94(2):501-509

35. Olsen SF et al (2007) Milk consumption during pregnancy is associated with increased infant size at birth: prospective cohort study. Am J Clin Nutr 86(4):1104-1110

36. Crozier SR et al (2017) Nausea and vomiting in early pregnancy: effects on food intake and diet quality. Matern Child Nutr 13(4):e12389

37. Wood F et al (2010) A question of balance: a qualitative study of mothers' interpretations of dietary recommendations. Ann Fam Med 8(1):51-57

38. Raberg Kjollesdal MK et al (2010) Barriers to healthy eating among Norwegian-Pakistani women participating in a culturally adapted intervention. Scand J Public Health 38(5 Suppl):52-59

39. Burnett-Hartman AN et al (2009) Supplement use contributes to meeting recommended dietary intakes for calcium, magnesium, and vitamin $\mathrm{C}$ in four ethnicities of middle-aged and older Americans: the multi-ethnic study of atherosclerosis. J Am Diet Assoc 109(3):422-429

40. Morisset AS et al (2016) Rankings of iron, vitamin D, and calcium intakes in relation to maternal characteristics of pregnant Canadian women. Appl Physiol Nutr Metab 41(7):749-757

41. Wolf HT et al (2017) Multivitamin use and adverse birth outcomes in high-income countries: a systematic review and meta-analysis. Am J Obstet Gynecol 217:404-412

42. Meertens LJE, Scheepers HCJ, Willemse JPMM, Spaanderman MEA, Smits LJM (2017) Should women be advised to use calcium supplements during pregnancy? A decision analysis. Matern Child Nutr 14:e12479

43. Centraal Bureau voor de Statistiek (2016) Jonge vrouwen vaker hoogopgeleid dan jonge mannen (Young women more often highly educated than young men). https://www.cbs.nl/nl-nl/nieuw s/2016/10/jonge-vrouwen-vaker-hoogopgeleid-dan-jonge-manne n. Assessed 26 May 2017

44. Hjartaker A, Andersen LF, Lund E (2007) Comparison of diet measures from a food-frequency questionnaire with measures from repeated 24-hour dietary recalls. The Norwegian women and cancer study. Public Health Nutr 10(10):1094-1103

45. Molag ML, de Vries JH, Duif N, Ocke MC, Dagnelie PC, Goldbohm RA et al (2010) Selecting informative food items for compiling food-frequencyquestionnaires: comparison of procedures. Br J Nutr 104(3):446-456

46. Kummeling I et al (2005) Etiology of atopy in infancy: the KOALA birth cohort study. Pediatr Allergy Immunol 16(8):679-684

47. Gomez MF et al (2015) Use of micronutrient supplements among pregnant women in Alberta: results from the Alberta pregnancy outcomes and nutrition (APrON) cohort. Matern Child Nutr 11(4):497-510 